\title{
New Results on Impulsive Functional Differential Equations with Infinite Delays
}

\author{
Jie Yang ${ }^{1}$ and Bing Xie ${ }^{2}$ \\ ${ }^{1}$ School of Mathematical Sciences, Shandong Normal University, Jinan 250014, China \\ ${ }^{2}$ Department of Mathematics, Shandong University, Weihai, Shandong 264209, China \\ Correspondence should be addressed to Jie Yang; jiey@sdnu.edu.cn
}

Received 28 January 2013; Accepted 6 April 2013

Academic Editor: Jinde Cao

Copyright (c) 2013 J. Yang and B. Xie. This is an open access article distributed under the Creative Commons Attribution License, which permits unrestricted use, distribution, and reproduction in any medium, provided the original work is properly cited.

We investigate the stability for a class of impulsive functional differential equations with infinite delays by using Lyapunov functions and Razumikhin-technique. Some new Razumikhin-type theorems on stability are obtained, which shows that impulses do contribute to the system's stability behavior. An example is also given to illustrate the importance of our results.

\section{Introduction}

Impulsive differential equations have attracted the interest of many researchers in recent years. It arises naturally from a wide variety of applications such as orbital transfer of satellite, ecosystems management, and threshold theory in biology. There has been a significant development in the theory of impulsive differential equations in the past several years ago, and various interesting results have been reported; see [1-4]. Recently, systems with impulses and time delay have received significant attention [5-16]. In fact, the system stability and convergence properties are strongly affected by time delays, which are often encountered in many industrial and natural processes due to measurement and computational delays, transmission, and transport lags. In $[5,6,8]$, the authors considered the stability of impulsive differential equations with finite delay and got some results. In [7], by using Lyapunov functions and Razumikhin technique, some Razumikhin-type theorems on stability are obtained for a class of impulsive functional differential equations with infinite-delay. However, not much has been developed in the direction of the stability theory of impulsive functional differential systems, especially for infinite delays impulsive functional differential systems. As we know, there are a number of difficulties that one must face in developing the corresponding theory of impulsive functional differential systems with infinite-delay; for example, the interval $(-\infty, \sigma]$ is not compact, and the images of a solution map of closed and bounded sets in $C\left((-\infty, 0], R_{n}\right)$ space may not be compact. Therefore, it is an interesting and complicated problem to study the stability theory for impulsive functional differential systems with infinite delays.

In the present paper, we will consider the stability of impulsive infinite-delay differential equations by using Lyapunov functions and the Razumikhin technique, we get some new results. The effect of delay and impulses which do contribute to the equations's stability properties will be shown in this paper.

The rest of this paper is organized as follows. In Section 2, we introduce some notations and definitions. In Section 3, we get some criteria for uniform stability and uniform asymptotic stability for impulsive infinite-delay differential equations, and an example is given to illustrate our results. Finally, concluding remarks are given in Section 4 .

\section{Preliminaries}

Let $R$ denote the set of real numbers, $R_{+}$the set of nonnegative real numbers, and $R^{n}$ the $n$-dimensional real space equipped with the Euclidean norm $\|\cdot\|$. For any $t \geq t_{0} \geq 0>\alpha \geq-\infty$, let $f(t, x(s))$ where $s \in[t+\alpha, t]$ or $f(t, x(\cdot))$ be a Volterra-type functional. In the case when $\alpha=-\infty$, the interval $[t+\alpha, t]$ is understood to be replaced by $(-\infty, t]$. 
We consider the impulsive functional differential equations

$$
\begin{aligned}
x^{\prime}(t)= & f(t, x(\cdot)), \quad t \geq t_{0}, \quad t \neq t_{k}, \\
\left.\Delta x\right|_{t=t_{k}} & =x\left(t_{k}\right)-x\left(t_{k}^{-}\right) \\
& =I_{k}\left(x\left(t_{k}^{-}\right)\right), \quad k=1,2, \ldots,
\end{aligned}
$$

where the impulse times $t_{k}$ satisfy $0 \leq t_{0}<t_{1}<\cdots<$ $t_{k}<\cdots, \lim _{k \rightarrow+\infty} t_{k}=+\infty$ and $x^{\prime}$ denotes the right-hand derivative of $x . f \in C\left(\left[t_{k-1}, t_{k}\right) \times C, R^{n}\right), f(t, 0)=0 . C$ is an open set in $\operatorname{PC}\left([\alpha, 0], R^{n}\right)$, where $\operatorname{PC}\left([\alpha, 0], R^{n}\right)=\{\psi:$ $[\alpha, 0] \rightarrow R^{n}$ is continuous everywhere except at finite number of points $t_{k}$, at which $\psi\left(t_{k}^{+}\right)$and $\psi\left(t_{k}^{-}\right)$exist and $\left.\psi\left(t_{k}^{+}\right)=\psi\left(t_{k}\right)\right\}$. For each $k=1,2, \ldots, I(t, x) \in C\left(\left[t_{0}, \infty\right) \times\right.$ $\left.R^{n}, R^{n}\right), I\left(t_{k}, 0\right)=0$.

For any $\rho>0$, there exists a $\rho_{1}>0\left(0<\rho_{1}<\rho\right)$ such that $x \in S\left(\rho_{1}\right)$ implies that $x+I\left(t_{k}, x\right) \in S(\rho)$, where $S(\rho)=\{x$ : $\left.\|x\|<\rho, x \in R^{n}\right\}$.

Define $\operatorname{PCB}(t)=\{x \in C: x$ is bounded $\}$. For $\psi \in$ $\operatorname{PCB}(t)$, the norm of $\psi$ is defined by $\|\psi\|=\sup _{\alpha \leq \theta \leq 0}|\psi(\theta)|$. For any $\sigma \geq 0$, let $\operatorname{PCB}_{\delta}(\sigma)=\{\psi \in \operatorname{PCB}(\sigma):\|\psi\|<\delta\}$.

For any given $\sigma \geq t_{0}$, the initial condition for system (1) is given by

$$
x_{\sigma}=\phi \text {, }
$$

where $\phi \in \mathrm{PC}\left([\alpha, 0], R^{n}\right)$.

We assume that the solution for the initial problems, (1)(2) does exist and is unique which will be written in the form $x(t, \sigma, \phi)$; see $[4,10]$. Since $f(t, 0)=0, I\left(t_{k}, 0\right)=0, k=$ $1,2, \ldots$, then $x(t)=0$ is a solution of (1)-(2), which is called the trivial solution. In this paper, we always assume that the solution $x(t, \sigma, \phi)$ of (1)-(2) can be continued to $\infty$ from the right of $\sigma$.

For convenience, we also have the following classes in later sections:

$$
\begin{aligned}
& K_{1}=\left\{a \in C\left(R_{+}, R_{+}\right) \mid a(0)=0 \text { and } a(s)>0\right. \text { for } \\
& s>0\} ; \\
& K_{2}=\left\{a \in C\left(R_{+}, R_{+}\right) \mid a(0)=0 \text { and } a(s)>0 \text { for } s>0\right. \\
& \text { and } a \text { is nondecreasing in } s\} ; \\
& \Delta V\left(t_{k}, \psi(0)\right) \quad=\quad V\left(t_{k}, \psi(0)+I_{k}\left(t_{k}, \psi\right)\right)- \\
& V\left(t_{k}^{-}, \psi(0)\right), k=1,2, \ldots ; \\
& \Delta t_{k}=t_{k}-t_{k-1}, k=1,2, \ldots
\end{aligned}
$$

We introduce some definitions as follows.

Definition 1 (see [4]). The function $V:[\alpha, \infty) \times C \rightarrow R_{+}$ belongs to class $v_{0}$ if

$\left(A_{1}\right) V$ is continuous on each of the sets $\left[t_{k-1}, t_{k}\right) \times C$ and $\lim _{(t, \varphi) \rightarrow\left(t_{k}^{-}, \psi\right)} V(t, \varphi)=V\left(t_{k}^{-}, \psi\right)$ exists;

$\left(A_{2}\right) V(t, x)$ is locally Lipschitzian in $x$ and $V(t, 0) \equiv 0$.
Definition 2 (see [4]). Let $V \in v_{0}$, for any $(t, \psi) \in\left[t_{k-1}, t_{k}\right) \times$ $C$, the upper right-hand Dini derivative of $V(t, x)$ along the solution of (1)-(2) is defined by

$$
\begin{aligned}
& D^{+} V(t, \psi(0)) \\
& \quad=\frac{\limsup _{h \rightarrow 0^{+}}\{V(t+h, \psi(0)+h f(t, \psi))-V(t, \psi(0))\}}{h} .
\end{aligned}
$$

Similarly, we can define $D^{-} V(t, \psi(0)), D_{-} V(t, \psi(0)$, $D_{+} V(t, \psi(0))$. If $V \in C^{\prime}$, then $D V(t, \psi(0))=\dot{V}(t, \psi(0))$, where $D$ is any of the four Dini derivatives.

For $V \in v_{0},(t, \psi) \in\left[t_{k-1}, t_{k}\right) \times C$, the upper righthand Dini derivative of $\dot{V}(t, x)$ along the solution of (1)-(2) is defined by

$$
\begin{aligned}
& D^{+} \dot{V}(t, \psi(0)) \\
& \quad=\frac{\limsup _{h \rightarrow 0^{+}}\{\dot{V}(t+h, \psi(0)+h f(t, \psi))-\dot{V}(t, \psi(0))\}}{h} .
\end{aligned}
$$

Similarly, we can define $D^{-} \dot{V}(t, \psi(0))$. If $V \in C^{\prime \prime}$, then these are simply the second derivative of $V$.

Definition 3 (see [4]). Assume $x(t)=x(t, \sigma, \phi)$ to be the solution of (1)-(2) through $(\sigma, \phi)$. Then, the zero solution of (1)-(2) is said to be

(1) uniformly stable, if for any $\varepsilon>0$ and $\sigma \geq t_{0}$, there exists a $\delta=\delta(\varepsilon)>0$ such that $\phi \in \operatorname{PCB}_{\delta}(\sigma)$ implies $\|x(t)\|<\varepsilon, t \geq \sigma$.

(2) uniformly asymptotically stable, if it is uniformly stable, and there exists a $\delta>0$ such that for any $\varepsilon>$ $0, \sigma \geq t_{0}$, there is a $T=T(\varepsilon)>0$ such that $\phi \in \mathrm{PCB}_{\delta}(\sigma)$ implies $\|x(t)\|<\varepsilon, t \geq \sigma+T$.

\section{Main Results}

Theorem 4. Assume that there exist functions $w_{i} \in K_{1}, g \in$ $K_{2}, c_{i}, p, q \in C\left(R_{+}, R_{+}\right), V(t, x) \in v_{0}, i=1,2$, and constants $m>1$, such that the following conditions hold:

(i) $w_{1}(\|x\|) \leq V(t, x) \leq w_{2}(\|x\|),(t, x) \in[\alpha, \infty) \times S(\rho)$;

(ii) for any $\sigma \geq t_{0}$ and $\psi \in P C([\alpha, 0], S(\rho))$, if $V(t, \psi(0)) \geq$ $m^{-2} g(V(t+\theta, \psi(\theta))), \max \{\alpha,-q(V(t))\} \leq \theta \leq$ $0, t \neq t_{k}$, then

$$
D^{+} V(t, \psi(0)) \leq p(t) c_{1}(V(t, \psi(0))),
$$

where $s / m \leq g(s)<s$ for any $s>0$;

(iii) for all $(t, \psi(0)) \in\left(t_{k-1}, t_{k}\right) \times P C\left([\alpha, 0], S\left(\rho_{1}\right)\right)$,

$$
D^{-} \dot{V}(t, \psi(0)) \geq 0 \text {. }
$$

Also, for all $\left(t_{k}, \psi\right) \in R_{+} \times P C\left([\alpha, 0], S\left(\rho_{1}\right)\right)$,

$$
\Delta t_{k} \dot{V}\left(t_{k}^{-}, \psi(0)\right)+\Delta V\left(t_{k}, \psi(0)\right) \leq-\mu_{k} c_{2}\left(V\left(t_{k}, \psi(0)\right)\right) \text {, }
$$

where $c_{2}(s) \leq s c_{2}^{\prime}(s), s>0, \mu_{k}$ satisfies $\liminf _{k \rightarrow \infty}$ $\mu_{k} \geq 2 \cdot \sup _{s>0}\left(s / c_{2}\left(m^{-1} \cdot s\right)\right)$; 
(iv) there exist constants $M_{1}, M_{2}>0$ such that the following inequalities hold:

$$
\begin{gathered}
\sup _{t \geq 0} \int_{t}^{t+\tau} p(s) d s=M_{1}<\infty, \\
\inf _{s>0} \int_{g(s)}^{s} \frac{d t}{c_{1}(t)}=M_{2}>M_{1}, \\
\text { where } \tau=\max _{k \geq 1}\left\{t_{k}-t_{k-1}\right\}<\infty .
\end{gathered}
$$

Then, the zero solution of (1)-(2) is uniformly asymptotically stable.

Proof. Condition (i) implies that $w_{1}(s) \leq w_{2}(s)$ for $s \in[0, \rho]$. So let $W_{1}$ and $W_{2}$ be continuous, strictly increasing functions satisfying $W_{1}(s) \leq w_{1}(s) \leq w_{2}(s) \leq W_{2}(s)$ for all $s \in[0, \rho]$. Then

$$
W_{1}(\|x\|) \leq V(t, x) \leq W_{2}(\|x\|), \quad(t, x) \in[\alpha, \infty) \times S(\rho) .
$$

We first show uniform stability.

For any $\varepsilon>0\left(<\rho_{1}\right)$, one may choose a $\delta=\delta(\varepsilon)>0$ such that $W_{2}(\delta) \leq g\left(W_{1}(\varepsilon)\right)$. Let $x(t)=x(t, \sigma, \phi)$ be a solution of (1)-(2) through $(\sigma, \phi), \sigma \geq t_{0}$. For any $\phi \in \operatorname{PCB}_{\delta}(\sigma)$, we will prove that $\|x(t)\|<\varepsilon, t \geq \sigma$.

For convenience, let $V(t)=V(t, x(t))$. Suppose that $\sigma \in$ $\left[t_{l-1}, t_{l}\right), l \in Z_{+}$. First, for $\sigma+\alpha \leq t \leq \sigma$, we have

$$
W_{1}(\|x\|) \& \leq V(t)<W_{2}(\delta) \leq g\left(W_{1}(\varepsilon)\right)<W_{1}(\varepsilon) .
$$

So, $\|x(t)\|<\varepsilon<\rho_{1}, t \in[\sigma+\alpha, \sigma]$.

Next, we claim that

$$
V(t)<W_{1}(\varepsilon), \quad t \in\left[\sigma, t_{l}\right) .
$$

Suppose on the contrary that there exists some $t \in\left[\sigma, t_{l}\right)$ such that $V(t) \geq W_{1}(\varepsilon)$. Since $V(\sigma)<W_{1}(\varepsilon)$, we can define $\widehat{t}=$ $\inf \left\{t \in\left[\sigma, t_{l}\right) \mid V(t) \geq W_{1}(\varepsilon)\right\}$. Thus, $\widehat{t} \in\left(\sigma, t_{l}\right), V(\widehat{t})=$ $W_{1}(\varepsilon)$, and $V(t)<W_{1}(\varepsilon), t \in[\sigma, \hat{t})$. Also, from (10) we obtain

$$
V(t)<W_{1}(\varepsilon), \quad t \in[\sigma+\alpha, \widehat{t}) .
$$

On the other hand, note that $V(\widehat{t})=W_{1}(\varepsilon)>g\left(W_{1}(\varepsilon)\right)$ and $V(\sigma)<g\left(W_{1}(\varepsilon)\right)$ in view of (10), we can define $t^{*}=\sup \{t \in$ $\left.[\sigma, \widehat{t}] V(t) \leq g\left(W_{1}(\varepsilon)\right)\right\}$; it is obvious that $t^{*} \in[\sigma, \widehat{t}), V\left(t^{*}\right)=$ $g\left(W_{1}(\varepsilon)\right)$ and $V(t)>g\left(W_{1}(\varepsilon)\right)$ for $t \in\left(t^{*}, \widehat{t}\right]$. Therefore, combining (12), we have for $t \in\left(t^{*}, \widehat{t}\right)$

$$
V(t)>g\left(W_{1}(\varepsilon)\right)>g(V(t+\theta)), \quad \alpha \leq \theta \leq 0 ;
$$

that is,

$$
\begin{gathered}
V(t, \psi(0))>m^{-2} g(V(t+\theta, \psi(\theta))), \\
\max \{\alpha,-q(V(t))\} \leq \theta \leq 0 .
\end{gathered}
$$

By assumption (ii), (iv), we have

$$
\int_{V\left(t^{*}\right)}^{V(t)} \frac{d s}{c_{1}(s)}=\int_{g\left(W_{1}(\varepsilon)\right)}^{W_{1}(\varepsilon)} \frac{d s}{c_{1}(s)} \geq M_{2}>M_{1} .
$$

However, we also have

$$
\int_{V\left(t^{*}\right)}^{V(t)} \frac{d s}{c_{1}(s)} \leq \int_{t^{*}}^{\widehat{t}} p(s) d s<\int_{t^{*}}^{t^{*}+\tau} p(s) d s \leq M_{1},
$$

which is a contradiction. So, (11) holds.

Hence, $W_{1}(\|x\|) \leq V(t)<W_{1}(\varepsilon), t \in\left[\sigma, t_{l}\right)$ implies that $\left\|x\left(t_{l}^{-}\right)\right\|<\varepsilon<\rho_{1}$. Thus, $x\left(t_{l}\right) \in S(\rho)$.

On the other hand, from condition (iii), we note for $k=$ $1,2, \ldots$

$$
\begin{aligned}
V\left(t_{k}\right)-V\left(t_{k-1}\right) & =V\left(t_{k}\right)-V\left(t_{k}^{-}\right)+V\left(t_{k}^{-}\right)-V\left(t_{k-1}\right) \\
& =\Delta V\left(t_{k}\right)+\int_{t_{k-1}}^{t_{k}} \dot{V}(t) d t \\
& \leq \Delta V\left(t_{k}\right)+\Delta t_{k} \dot{V}\left(t_{k}^{-}\right) \\
& \leq-\mu_{k} \mathcal{C}_{2}\left(V\left(t_{k}\right)\right) \leq 0 .
\end{aligned}
$$

Hence, we obtain $V\left(t_{k}\right) \leq V\left(t_{k-1}\right), k=1,2, \ldots$ particularly, $V\left(t_{l}\right) \leq V\left(t_{l-1}\right)$. In view of (10), we get

$$
V\left(t_{l}\right) \leq V\left(t_{l-1}\right)<g\left(W_{1}(\varepsilon)\right)<W_{1}(\varepsilon) .
$$

Next, we claim that

$$
V(t)<W_{1}(\varepsilon), \quad t \in\left[t_{l}, t_{l+1}\right) .
$$

Suppose on the contrary that there exists some $t \in\left[t_{l}, t_{l+1}\right)$ such that $V(t) \geq W_{1}(\varepsilon)$. Then applying exactly the same argument as in the proof of (11) yields our desired contradiction.

By induction hypothesis, we may prove, in general, that for $t \in\left[t_{l+k}, t_{l+k+1}\right), k>0$,

$$
V(t)<W_{1}(\varepsilon)
$$

In view of condition (i), we obtain that $\|x(t)\|<\varepsilon, t \geq \sigma$. Therefore, we have proved that the solutions of (1)-(2) are uniformly stable.

Next, we claim that they are uniformly asymptotically stable. Since the zero solution of (1)-(2) is uniformly stable, for any given constant $H>0\left(<\rho_{1}\right)$, then there exists $\delta>0$ such that $\phi \in \operatorname{PCB}_{\delta}(\sigma)$ implies that $V(t)<W_{1}(H),\|x(t)\|<$ $\rho_{1}, t \geq \sigma$.

For any $\varepsilon \in(0, H)$, let

$$
\begin{gathered}
d<\min \left\{\widehat{d}, W_{1}(\varepsilon)\right\}, \\
\widehat{d}=\inf \left\{s-g(s) \mid m^{-1} W_{1}(\varepsilon) \leq s \leq W_{1}(H)\right\}, \\
h=\sup \left\{q(s) \mid m^{-1} W_{1}(\varepsilon) \leq s \leq W_{1}(H)\right\}, \\
n_{0}=\frac{W_{1}(H)}{2 \cdot \sup _{s>0}\left(s / c_{2}\left(m^{-1} s\right)\right) c_{2}\left(m^{-1} W_{1}(\varepsilon)\right)}+1 .
\end{gathered}
$$

From condition (iii), we get that there exists a $n_{1}>0$ such that for $k>n_{1}$,

$$
\mu_{k} \geq 2 \cdot \sup _{s>0} \frac{s}{c_{2}\left(m^{-1} \cdot s\right)}
$$


Choose a positive integer $N$ satisfying

$$
W_{1}(\varepsilon)+(N-1) d<W_{1}(H) \leq W_{1}(\varepsilon)+N d \text {, }
$$

and define $T=N\left(h+n_{0} \tau\right)+n_{1}$, we will prove that $\phi \epsilon$ $\operatorname{PCB}_{\delta}(\sigma)$ implies $\|x(t)\|<\varepsilon, t \geq \sigma+T$.

First, we prove that there exists $\widehat{t} \in\left[\sigma+h+n_{1}, \sigma+h+n_{1}+\right.$ $\left.n_{0} \tau\right]$ such that

$$
V(\widehat{t})<m^{-1}\left[W_{1}(\varepsilon)+(N-1) d\right] .
$$

Suppose on the contrary that for all $t \in\left[\sigma+h+n_{1}, \sigma+h+\right.$ $\left.n_{1}+n_{0} \tau\right]$

$$
V(t) \geq m^{-1}\left[W_{1}(\varepsilon)+(N-1) d\right] \geq m^{-1} W_{1}(\varepsilon) .
$$

Let $t_{k_{1}}=\min \left\{t_{k}: t_{k} \geq \sigma+h+n_{1}\right\}$, from (17), we get

$$
\begin{gathered}
V\left(t_{k_{1}}\right)-V\left(t_{k_{1}-1}\right) \leq-\mu_{k_{1}} c_{2}\left(V\left(t_{k_{1}}\right)\right) \\
\leq-\mu_{k_{1}} c_{2}\left(m^{-1} W_{1}(\varepsilon)\right), \\
V\left(t_{k_{1}+1}\right)-V\left(t_{k_{1}}\right) \leq-\mu_{k_{1}+1} c_{2}\left(m^{-1} W_{1}(\varepsilon)\right), \\
\vdots \\
V\left(t_{k_{1}+n_{0}}\right)-V\left(t_{k_{1}+n_{0}-1}\right) \leq-\mu_{k_{1}+n_{0}} c_{2}\left(m^{-1} W_{1}(\varepsilon)\right),
\end{gathered}
$$

In general, combining (22), we deduce that

$$
\begin{aligned}
V\left(t_{k_{1}+n_{0}}\right) \leq & V\left(t_{k_{1}-1}\right)-\sum_{s=0}^{n_{0}} \mu_{k_{1}+s} c_{2}\left(m^{-1} W_{1}(\varepsilon)\right) \\
\leq & W_{1}(H)-2\left(n_{0}+1\right) \\
& \cdot \sup _{s>0} \frac{s}{c_{2}\left(m^{-1} s\right)} c_{2}\left(m^{-1} W_{1}(\varepsilon)\right) \\
= & -4 \cdot \sup _{s>0} \frac{s}{c_{2}\left(m^{-1} s\right)} c_{2}\left(m^{-1} W_{1}(\varepsilon)\right)<0,
\end{aligned}
$$

which is a contradiction. So, (24) holds.

Suppose $\widehat{t} \in\left[t_{l-1}, t_{l}\right), l>1$. Furthermore, we can prove that for $t \in\left[\widehat{t}, t_{l}\right)$

$$
V(t)<W_{1}(\varepsilon)+(N-1) d .
$$

Suppose this assertion is false, then there exists some $t \in\left[\widehat{t}, t_{l}\right)$ such that $V(t) \geq W_{1}(\varepsilon)+(N-1) d$. Since $V(\widehat{t})<m^{-1}\left[W_{1}(\varepsilon)+\right.$ $(N-1) d]<W_{1}(\varepsilon)+(N-1) d$, so define

$$
t^{*}=\inf \left\{t \in\left[\widehat{t}, t_{l}\right) \mid V(t) \geq W_{1}(\varepsilon)+(N-1) d\right\}
$$

then $t^{*} \in\left(\widehat{t}, t_{l}\right), V\left(t^{*}\right)=W_{1}(\varepsilon)+(N-1) d$ and $V(t)<W_{1}(\varepsilon)+$ $(N-1) d, t \in\left(\widehat{t}, t^{*}\right)$. Note that

$$
\begin{gathered}
V\left(t^{*}\right)=W_{1}(\varepsilon)+(N-1) d>g\left(W_{1}(\varepsilon)+(N-1) d\right), \\
V(\widehat{t})<m^{-1}\left[W_{1}(\varepsilon)+(N-1) d\right]<g\left(W_{1}(\varepsilon)+(N-1) d\right) ;
\end{gathered}
$$

thus, we can define

$$
\bar{t}=\sup \left\{t \in\left[\widehat{t}, t^{*}\right] \mid V(t) \leq g\left(W_{1}(\varepsilon)+(N-1) d\right)\right\},
$$

then $\bar{t} \in\left[\widehat{t}, t^{*}\right), V(\bar{t})=g\left(W_{1}(\varepsilon)+(N-1) d\right)$ and $V(t)>$ $g\left(W_{1}(\varepsilon)+(N-1) d\right)$ for $t \in\left(\bar{t}, t^{*}\right]$.

Hence, we get for $t \in\left(\bar{t}, t^{*}\right]$

$$
\begin{aligned}
V(t) & >g\left(W_{1}(\varepsilon)+(N-1) d\right) \\
& \geq m^{-1}\left[W_{1}(\varepsilon)+(N-1) d\right] \\
& \geq m^{-1} W_{1}(\varepsilon),
\end{aligned}
$$

which implies that for $t \in\left(\bar{t}, t^{*}\right]$

$$
\begin{aligned}
V(t) & \geq g(V(t))+d \geq m^{-1} V(t)+d \\
& >\frac{m V(t)}{m^{2}}+\frac{d}{m^{2}} \geq \frac{W_{1}(\varepsilon)+N d}{m^{2}} \\
& \geq \frac{W_{1}(H)}{m^{2}} \geq \frac{V(s)}{m^{2}}>\frac{g(V(s))}{m^{2}}, \quad t+\alpha<s \leq t .
\end{aligned}
$$

Thus, $V(t) \geq\left(1 / m^{2}\right) g(V(t+\theta, \psi(\theta))), \max \{\alpha,-q(V(t))\} \leq$ $\theta \leq 0$.

By assumption, (ii), (iv), we have for $t \in\left(\bar{t}, t^{*}\right)$,

$$
\int_{V(\bar{t})}^{V\left(t^{*}\right)} \frac{d s}{c_{1}(s)}=\int_{g\left(W_{1}(\varepsilon)+(N-1) d\right)}^{W_{1}(\varepsilon)+(N-1) d} \frac{d s}{c_{1}(s)} \geq M_{2}>M_{1} .
$$

However, we also have

$$
\int_{V(\bar{t})}^{V\left(t^{*}\right)} \frac{d s}{c_{1}(s)}<\int_{\bar{t}}^{t^{*}} p(s) d s<\int_{\bar{t}}^{\bar{t}+\tau} p(s) d s<M_{1}
$$

which is a contradiction. So, (28) holds.

On the other hand, it is easy to prove that the functions $s / c_{2}\left(m^{-1} s\right)$ are nonincreasing for $s \in(0,+\infty)$ in view of condition $c_{2}(s) \leq s c_{2}^{\prime}(s)$ for any $s>0$.

Hence, the following inequalities hold: for $k>n_{1}$,

$$
\begin{aligned}
\frac{W_{1}(\varepsilon)+(N-i) d}{c_{2}\left(m^{-1}\left(W_{1}(\varepsilon)+(N-i-1) d\right)\right)} & \leq \frac{W_{1}(\varepsilon)+d}{c_{2}\left(m^{-1} W_{1}(\varepsilon)\right)} \\
& <\frac{2 W_{1}(\varepsilon)}{c_{2}\left(m^{-1} W_{1}(\varepsilon)\right)} \\
& \leq \mu_{k}, \quad i=1,2, \ldots, N-1 .
\end{aligned}
$$

Next, we claim that

$$
V\left(t_{l}\right)<m^{-1}\left[W_{1}(\varepsilon)+(N-1) d\right] .
$$

Or else, then $V\left(t_{l}\right) \geq m^{-1}\left[W_{1}(\varepsilon)+(N-1) d\right]$; from (17), we get

$$
\begin{aligned}
V\left(t_{l}\right)-V\left(t_{l-1}\right) & \leq-\mu_{l} \mathcal{c}_{2}\left(V\left(t_{l}\right)\right) \\
& \leq-\mu_{l} \mathcal{c}_{2}\left(m^{-1}\left[W_{1}(\varepsilon)+(N-1) d\right]\right)
\end{aligned}
$$


Considering (36), it holds that

$$
\begin{aligned}
V\left(t_{l}\right) \leq & V\left(t_{l-1}\right)-\mu_{l} c_{2}\left(m^{-1}\left[W_{1}(\varepsilon)+(N-1) d\right]\right) \\
\leq & W_{1}(H)-\mu_{l} c_{2}\left(m^{-1}\left[W_{1}(\varepsilon)+(N-1) d\right]\right) \\
\leq & W_{1}(\varepsilon)+N d-\mu_{l} c_{2}\left(m^{-1}\left[W_{1}(\varepsilon)+(N-1) d\right]\right) \\
\leq & c_{2}\left(m^{-1}\left[W_{1}(\varepsilon)+(N-1) d\right]\right) \\
& \times\left\{\frac{W_{1}(\varepsilon)+N d}{c_{2}\left(m^{-1}\left[W_{1}(\varepsilon)+(N-1) d\right]\right)}-\mu_{l}\right\} \\
< & 0,
\end{aligned}
$$

which is a contradiction and (37) holds.

Next, we can prove that for $t \in\left[t_{l}, t_{l+1}\right)$

$$
V(t)<W_{1}(\varepsilon)+(N-1) d .
$$

Suppose that this assertion is false, then there exists some $t \epsilon$ $\left[\widehat{t}, t_{l}\right)$ such that $V(t) \geq W_{1}(\varepsilon)+(N-1) d$. Then applying exactly the same argument as in the proof of (24) and (28) yields our desired contradiction. Here, we omit it.

By induction hypothesis, we may prove, for $t \in$ $\left[t_{l+k}, t_{l+k+1}\right), k=1,2, \ldots$,

$$
V(t)<W_{1}(\varepsilon)+(N-1) d ;
$$

that is,

$$
V(t)<W_{1}(\varepsilon)+(N-1) d, \quad t \geq \widehat{t} .
$$

Hence, we obtain

$$
V(t)<W_{1}(\varepsilon)+(N-1) d, \quad t \geq \sigma+h+n_{1}+n_{0} \tau .
$$

Next, we prove that there exists $\widehat{t}_{2} \in\left[\sigma+2 h+n_{1}+n_{0} \tau, \sigma+\right.$ $\left.2 h+n_{1}+2 n_{0} \tau\right]$ such that

$$
V\left(\widehat{t}_{2}\right)<m^{-1}\left[W_{1}(\varepsilon)+(N-2) d\right] .
$$

Suppose that for all $t \in\left[\sigma+2 h+n_{1}+n_{0} \tau, \sigma+2 h+n_{1}+2 n_{0} \tau\right]$,

$$
V(t) \geq m^{-1}\left[W_{1}(\varepsilon)+(N-2) d\right] \geq m^{-1} W_{1}(\varepsilon) .
$$

Using the same argument as in the proof of (24), we get

$$
\begin{aligned}
V\left(t_{k_{2}+n_{0}}\right) \leq & V\left(t_{k_{2}-1}\right)-\sum_{s=0}^{n_{0}} \mu_{k_{2}+s} c_{2}\left(m^{-1} W_{1}(\varepsilon)\right) \\
\leq & W_{1}(H)-2\left(n_{0}+1\right) \\
& \cdot \sup _{s>0} \frac{s}{c_{2}\left(m^{-1} s\right)} c_{2}\left(m^{-1} W_{1}(\varepsilon)\right) \\
= & -4 \cdot \sup _{s>0} \frac{s}{c_{2}\left(m^{-1} s\right)} c_{2}\left(m^{-1} W_{1}(\varepsilon)\right)<0,
\end{aligned}
$$

where $t_{k_{2}}=\min \left\{t_{k}: k \geq \sigma+2 h+n_{1}+n_{0} \tau\right\}$.

This is a contradiction. So, (44) holds.
Suppose $\widehat{t}_{2} \in\left[t_{k-1}, t_{k}\right), k>l$. Furthermore, we claim that for $t \in\left[\widehat{t}_{2}, t_{k}\right)$

$$
V(t)<W_{1}(\varepsilon)+(N-2) d .
$$

Suppose on the contrary, that there exists some $t \in\left[\widehat{t}_{2}, t_{k}\right)$ such that $V(t) \geq W_{1}(\varepsilon)+(N-2) d$. We define

$$
t^{\star}=\inf \left\{t \in\left[\widehat{t}_{2}, t_{k}\right) \mid V(t) \geq W_{1}(\varepsilon)+(N-2) d\right\},
$$

since $V\left(\hat{t}_{2}\right)<m^{-1}\left[W_{1}(\varepsilon)+(N-2) d\right]<W_{1}(\varepsilon)+(N-2) d$ in view of (44). Thus, $t^{\star} \in\left(\hat{t}_{2}, t_{k}\right), V\left(t^{\star}\right)=W_{1}(\varepsilon)+(N-2) d$ and $V(t)<W_{1}(\varepsilon)+(N-2) d, t \in\left(\widehat{t}_{2}, t^{\star}\right)$. Note that

$$
\begin{gathered}
V\left(t^{\star}\right)=W_{1}(\varepsilon)+(N-2) d>g\left(W_{1}(\varepsilon)+(N-2) d\right), \\
V\left(\widehat{t}_{2}\right)<m^{-1}\left[W_{1}(\varepsilon)+(N-2) d\right]<g\left(W_{1}(\varepsilon)+(N-2) d\right) ;
\end{gathered}
$$

furthermore, we can define

$$
\tilde{t}=\sup \left\{t \in\left[\widehat{t}_{2}, t^{\star}\right] \mid V(t) \leq g\left(W_{1}(\varepsilon)+(N-2) d\right)\right\},
$$

then $\widetilde{t} \in\left[\widehat{t}_{2}, t^{\star}\right), V(\widetilde{t})=g\left(W_{1}(\varepsilon)+(N-2) d\right)$ and $V(t)>$ $g\left(W_{1}(\varepsilon)+(N-2) d\right)$ for $t \in\left(\widetilde{t}, t^{\star}\right]$.

Hence, we get for $t \in\left(\widetilde{t}, t^{\star}\right]$

$$
\begin{aligned}
V(t) & >g\left(W_{1}(\varepsilon)+(N-2) d\right) \\
& \geq m^{-1}\left[W_{1}(\varepsilon)+(N-2) d\right] \\
& \geq m^{-1} W_{1}(\varepsilon) ;
\end{aligned}
$$

considering the definition of $d$ and (43), we get for $t \in\left(\widetilde{t}, t^{\star}\right]$

$$
\begin{aligned}
V(t) & \geq g(V(t))+d \geq m^{-1} V(t)+d \\
& >\frac{m V(t)}{m^{2}}+\frac{d}{m^{2}} \geq \frac{W_{1}(\varepsilon)+(N-1) d}{m^{2}} \\
& \geq \frac{V(s)}{m^{2}}>\frac{g(V(s))}{m^{2}}, \quad t-h<s \leq t .
\end{aligned}
$$

Thus, $V(t) \geq\left(1 / m^{2}\right) g(V(t+\theta, \psi(\theta))), \max \{\alpha,-q(V(t))\} \leq$ $\theta \leq 0$.

Using assumptions (ii), (iv), we have

$$
\int_{V(\tilde{t})}^{V\left(t^{\star}\right)} \frac{d s}{c_{1}(s)}=\int_{g\left(W_{1}(\varepsilon)+(N-2) d\right)}^{W_{1}(\varepsilon)+(N-2) d} \frac{d s}{c_{1}(s)} \geq M_{2}>M_{1} .
$$

However,

$$
\int_{V(\tilde{t})}^{V\left(t^{\star}\right)} \frac{d s}{c_{1}(s)}<\int_{\tilde{t}}^{t^{\star}} p(s) d s<\int_{\tilde{t}}^{\tilde{t}+\tau} p(s) d s<M_{1}
$$

giving us a contradiction. So, (47) holds.

Next, we claim that

$$
\begin{gathered}
V\left(t_{l}\right)<m^{-1}\left[W_{1}(\varepsilon)+(N-1) d\right], \\
V(t)<W_{1}(\varepsilon)+(N-1) d, \quad t \in\left[t_{l}, t_{l+1}\right),
\end{gathered}
$$


whose arguments are the same as was employed in the proof of (36), (37). there we omit it.

Repeating this process, it is easy to check that

$$
V(t)<W_{1}(\varepsilon)+(N-2) d, \quad t \geq \sigma+2 h+n_{1}+2 n_{0} \tau .
$$

By induction hypothesis, we have

$$
V(t) \leq W_{1}(\varepsilon)+(N-i) d, \quad t \geq \sigma+i h+n_{1}+i n_{0} \tau .
$$

Let $i=N$, then for $t \geq \sigma+N\left(h+n_{0} \tau\right)+n_{1}$,

$$
V(t)<W_{1}(\varepsilon)
$$

Therefore, we arrive at $\|x(t)\|<\varepsilon, t \geq T$. The proof of Theorem 4 is complete.

Corollary 5. Assume that there exist functions $w_{i} \in K_{1}, g \in$ $K_{2}, c, p \in C\left(R_{+}, R_{+}\right), V(t, x) \in v_{0}, i=1,2$, and constants $m>1$, such that the following conditions hold:

(i) $w_{1}(\|x\|) \leq V(t, x) \leq w_{2}(\|x\|),(t, x) \in[\alpha, \infty) \times S(\rho)$;

(ii) for any $\sigma \geq t_{0}$ and $\psi \in P C([\alpha, 0], S(\rho))$, if $V(t, \psi(0)) \geq$ $g(V(t+\theta, \psi(\theta))), \alpha \leq \theta \leq 0, t \neq t_{k}$, then

$$
D^{+} V(t, \psi(0)) \leq p(t) c(V(t, \psi(0))),
$$

where $(s / m) \leq g(s)<s$ for any $s>0$;

(iii) for all $(t, \psi(0)) \in\left(t_{k-1}, t_{k}\right) \times P C\left([\alpha, 0], S\left(\rho_{1}\right)\right)$,

$$
D^{-} \dot{V}(t, \psi(0)) \geq 0 \text {. }
$$

Also, for all $\left(t_{k}, \psi\right) \in R_{+} \times P C\left([\alpha, 0], S\left(\rho_{1}\right)\right)$,

$$
\Delta t_{k} \dot{V}\left(t_{k}^{-}, \psi(0)\right)+\Delta V\left(t_{k}, \psi(0)\right) \leq 0 ;
$$

(iv) there exist constants $M_{1}, M_{2}>0$ such that the following inequalities hold:

$$
\begin{gathered}
\sup _{t \geq 0} \int_{t}^{t+\tau} p(s) d s=M_{1}<\infty \\
\inf _{s>0} \int_{g(s)}^{s} \frac{d t}{c(t)}=M_{2}>M_{1},
\end{gathered}
$$

where $\tau=\max _{k \geq 1}\left\{t_{k}-t_{k-1}\right\}<\infty$.

Then the zero solution of (1)-(2) is uniformly stable.

Theorem 4 has a dual result when $\dot{V}$ is nonincreasing on $\left(t_{k-1}, t_{k}\right)$. Here, we only give the results whose proof is very similar to the proof of Theorem 4 .

Theorem 6. Assume that there exist functions $w_{i} \in K_{1}, g \in$ $K_{2}, c_{i}, p, q \in C\left(R_{+}, R_{+}\right), V(t, x) \in v_{0}, i=1,2$, and constants $m>1$, such that the following conditions hold:

(i) $w_{1}(\|x\|) \leq V(t, x) \leq w_{2}(\|x\|),(t, x) \in[\alpha, \infty) \times S(\rho)$;

(ii) for any $\sigma \geq t_{0}$ and $\psi \in P C([\alpha, 0], S(\rho))$, if $V(t, \psi(0)) \geq m^{-2} g(V(t+\theta, \psi(\theta))), \max \{\alpha$, $-q(V(t))\} \leq \theta \leq 0, t \neq t_{k}$, then

$$
D^{+} V(t, \psi(0)) \leq p(t) c_{1}(V(t, \psi(0))),
$$

where $(s / m) \leq g(s)<s$ for any $s>0$; (iii) for all $(t, \psi(0)) \in\left(t_{k-1}, t_{k}\right) \times P C\left([\alpha, 0], S\left(\rho_{1}\right)\right)$,

$$
D^{-} \dot{V}(t, \psi(0)) \leq 0 .
$$

Also, for all $\left(t_{k}, \psi\right) \in R_{+} \times P C\left([\alpha, 0], S\left(\rho_{1}\right)\right)$,

$\Delta t_{k} \dot{V}\left(t_{k-1}^{-}, \psi(0)\right)+\Delta V\left(t_{k}, \psi(0)\right) \leq-\mu_{k} c_{2}\left(V\left(t_{k}, \psi(0)\right)\right)$,

where $c_{2}(s) \leq s c_{2}^{\prime}(s), s>0, \mu_{k}$ satisfies $\liminf _{k \rightarrow \infty}$ $\mu_{k} \geq 2 \cdot \sup _{s>0}\left(s / c_{2}\left(m^{-1} \cdot s\right)\right)$;

(iv) there exist constants $M_{1}, M_{2}>0$ such that the following inequalities hold:

$$
\begin{aligned}
& \sup _{t \geq 0} \int_{t}^{t+\tau} p(s) d s=M_{1}<\infty, \\
& \inf _{s>0} \int_{g(s)}^{s} \frac{d t}{c_{1}(t)}=M_{2}>M_{1},
\end{aligned}
$$

where $\tau=\max _{k \geq 1}\left\{t_{k}-t_{k-1}\right\}<\infty$.

Then the zero solution of (1)-(2) is uniformly asymptotically stable.

Example 7. Consider the impulsive delay differential equations:

$$
\begin{gathered}
x^{\prime}(t)=a x(t)-b \int_{-\infty}^{0} e^{s} x(t+s) d s, \quad t \geq 0, t \neq t_{k}, \\
\left.\Delta x\right|_{t=t_{k}}=I_{k}(x), \quad k=1,2, \ldots, \\
x_{0}=\phi>0,
\end{gathered}
$$

where $a \in(0,3], b \in(0,2],\left|x+I_{k}(x)\right| \leq \sqrt{\lambda} \cdot|x|, k=$ $1,2, \ldots, \lambda \in(0,1)$. For any given $\phi>0$, we always suppose that (68) has and only has positive solutions, and assume without loss of generality that $x(t)=x(t, 0, \phi)$ is a solutions of $(68)$ through $(0, \phi)$. Suppose that there exists $m>1$ such that the following inequalities hold:

$$
\tau<\min \left\{\frac{\ln m}{2(a-b \sqrt{m})}, \frac{1-\lambda-2 \lambda m}{2 a}\right\}, \quad a>2 b-1,
$$

where $\tau=\max _{k \geq 1}\left\{t_{k}-t_{k-1}\right\}<\infty$. Then, the zero solution of (68) is uniformly asymptotically stable.

In fact, let $V(t, x)=x^{2} / 2, g(s)=m^{-1} s(m>$ $1)$, and $c_{1}(s)=s$ then $V(t, x(t))>g(V(s, x(s))),-\infty \leq s \leq t$ implies that $\sqrt{m} \cdot|x(t)|>|x(s)|,-\infty \leq s \leq t$. Thus, for $t \neq t_{k}$

$$
\begin{aligned}
D^{+} V(t, x(\cdot)) & =x(t) x^{\prime}(t) \\
& =x(t)\left\{a x(t)-b \int_{-\infty}^{0} e^{s} x(t+s) d s\right\} \\
& \leq x^{2}(t)\left\{a-b \sqrt{m} \int_{-\infty}^{0} e^{s} d s\right\} \\
& =x^{2}(t)\{a-b \sqrt{m}\} \\
& =p(t) V(t, x(t))
\end{aligned}
$$

where $p(t)=2(a-b \sqrt{m})$. 
In view of condition (69), we note

$$
\begin{aligned}
\sup _{t \geq 0} \int_{t}^{t+\tau} p(s) d s & =2 \tau(a-b \sqrt{m})<\ln m \\
& =\inf _{s>0} \int_{g(s)}^{s} \frac{d t}{c_{1}(t)} .
\end{aligned}
$$

So, condition (iv) in Corollary 5 holds.

On the other hand, we have for $t \neq t_{k}$

$$
\begin{aligned}
D^{-} \dot{V}(t, x(\cdot))= & \left(x(t) x^{\prime}(t)\right)^{\prime} \\
= & x(t) x^{\prime \prime}(t)+\left(x^{\prime}(t)\right)^{2} \\
= & \left(x^{\prime}(t)\right)^{2} \\
& +x(t)\left(a x(t)-b \int_{-\infty}^{0} e^{s} x(t+s) d s\right)^{\prime} \\
= & \left(x^{\prime}(t)\right)^{2}+a x(t) x^{\prime}(t) \\
& -b x(t) \int_{-\infty}^{0} e^{s} x(t+s) d s-b x^{2}(t) \\
= & \left(x^{\prime}(t)\right)^{2}+a x(t) x^{\prime}(t)+a x^{2}(t) \\
& -x(t) x^{\prime}(t)-b x^{2}(t) \\
= & \left(x^{\prime}(t)\right)^{2}+(a-1) x(t) x^{\prime}(t) \\
& +(a-b) x^{2}(t) \\
\geq & \frac{3-a}{2}\left(x^{\prime}(t)\right)^{2}-\frac{\left(x^{\prime}(t)\right)^{2}+x^{2}(t)}{2}(a-1) \\
& +(a) x^{2}(t) \\
& \\
& \\
& \\
& \\
& \\
&
\end{aligned}
$$

in view of condition $a>2 b-1$. Also, considering $x(t)$ to be a positive solution of (68), we get

$$
\begin{aligned}
\Delta t_{k} & \dot{V}\left(t_{k}^{-}, \psi(0)\right)+\Delta V\left(t_{k}, \psi(0)\right) \\
& \leq a \tau x^{2}\left(t_{k}^{-}\right)+(\lambda-1) \frac{x^{2}\left(t_{k}^{-}\right)}{2} \\
& =(2 a \tau+\lambda-1) x^{2}\left(t_{k}^{-}\right) \frac{x^{2}\left(t_{k}^{-}\right)}{2} \\
& =(2 a \tau+\lambda-1) V\left(t_{k}^{-}\right) \\
& \leq-\frac{1-2 a \tau-\lambda}{\lambda} V\left(t_{k}\right) \\
& =-\mu_{k} c_{2}\left(V\left(t_{k}, \psi(0)\right)\right),
\end{aligned}
$$

where $c_{2}=s, \mu_{k}=(1-2 a \tau-\lambda) / \lambda$.
Note that

$$
\sup _{s>0} \frac{2 s}{c_{2}\left(m^{-1} \cdot s\right)}=2 m<\frac{1-2 a \tau-\lambda}{\lambda}=\mu_{k},
$$

in view of (69). So, the zero solution of (68) is uniformly stable by Corollary 5 .

Furthermore, choose $q(s)=-\ln (1-1 / m)$ (positive constants), which implies that $\int_{-q(s)}^{0} e^{s} d s=m^{-1}$. On the other hand, since $V(t, x(t))>m^{-2} g(V(s, x(s))), \max \{\alpha, t-$ $q(V(t))\} \leq s \leq t$, implying that $m^{3 / 2}|x(t)|>|x(s)|, \max \{\alpha, t-$ $q(V(t))\} \leq s \leq t$, then

$$
\begin{aligned}
&\left.D^{+} V\right|_{(68)}(t, x(\cdot)) \\
& \leq a x^{2}(t)-b x(t) \int_{-\infty}^{0} e^{s}|x(t+s)| d s \\
& \leq a x^{2}(t)-b x(t) \int_{-\infty}^{t} e^{s-t}|x(s)| d s \\
& \leq a x^{2}(t)-b x(t) \int_{t-q(V(t, x(\cdot)))}^{t} e^{s-t}|x(s)| d s \\
&-x(t) \int_{-\infty}^{t-q(V(t, x(\cdot)))} e^{s-t}|x(s)| d s \\
& \leq a x^{2}(t)-b x(t) \int_{t-q(V(t, x(\cdot)))}^{t} e^{s-t}|x(s)| d s \\
& \leq x^{2}(t)\left\{a-b m^{3 / 2} \int_{-q(V(t, x(\cdot)))}^{0} e^{s} d s\right\} \\
& \leq x^{2}(t)\{a-b \sqrt{m}\} \\
&= c(V(t, x(t))) p(t) .
\end{aligned}
$$

By Theorem 4, we obtain that if (69) holds, then the zero solution of (68) is uniformly asymptotically stable.

Remark 8. In fact, $x(t)=\phi(0) e^{t}$ is a positive solution of (68) through $(0, \phi)$ in the absence of impulses. It is obvious that the solution is unstable. However, the solution is uniformly asymptotically stable under proper impulses effect, which shows that impulses do contribute to the system's stability behavior.

\section{Conclusion}

In this work, we have considered the stability of impulsive infinite-delay differential systems. By using Lyapunov functions and the Razumikhin technique, we have obtained some new results. We can see that impulses and delay do contribute to the system's stability behavior.

\section{References}

[1] A. A. Soliman, "Stability criteria of impulsive differential systems," Applied Mathematics and Computation, vol. 134, no. 2-3, pp. 445-457, 2003. 
[2] D. D. Bainov and P. S. Simennov, Systems with Impulsive Effect Stability, Theory and Applications, Ellis Horwood, New York, NY, USA, 1989.

[3] T. Yang, Impulsive Systems and Control, Theory and Applications, Nova Science, Huntington, NY, USA, 2001.

[4] X. Fu, B. Yan, and Y. Liu, Introduction of Impulsive Differential Systems, Science Press, Beijing, China, 2005.

[5] Y. Xing and M. Han, "A new approach to stability of impulsive functional differential equations," Applied Mathematics and Computation, vol. 151, no. 3, pp. 835-847, 2004.

[6] J. Shen and J. Yan, "Razumikhin type stability theorems for impulsive functional-differential equations," Nonlinear Analysis. Theory, Methods \& Applications A, vol. 33, no. 5, pp. 519-531, 1998.

[7] Z. G. Luo and J. H. Shen, "Impulsive stabilization of functional differential equations with infinite delays," Applied Mathematics Letters, vol. 16, no. 5, pp. 695-701, 2003.

[8] Z. G. Luo and J. H. Shen, "New Razumikhin type theorems for impulsive functional differential equations," Applied Mathematics and Computation, vol. 125, no. 2-3, pp. 375-386, 2002.

[9] Q. Wang and X. Liu, "Exponential stability for impulsive delay differential equations by Razumikhin method," Journal of Mathematical Analysis and Applications, vol. 309, no. 2, pp. 462473, 2005.

[10] J. Shen, "Existence and uniqueness of solutions for a class of infinite delay functional differential equations with applications to impulsive differential equations," Journal of Huaihua Teachers College, vol. 15, pp. 45-451, 1996.

[11] Y. Zhang and J. Sun, "Stability of impulsive infinite delay differential equations," Applied Mathematics Letters, vol. 19, no. 10, pp. 1100-1106, 2006.

[12] Y. Zhang and J. Sun, "Stability of impulsive functional differential equations," Nonlinear Analysis. Theory, Methods \& Applications A, vol. 68, no. 12, pp. 3665-3678, 2008.

[13] X. Li, "New results on global exponential stabilization of impulsive functional differential equations with infinite delays or finite delays," Nonlinear Analysis. Real World Applications, vol. 11, no. 5, pp. 4194-4201, 2010.

[14] X. Li, "Further analysis on uniform stability of impulsive infinite delay differential equations," Applied Mathematics Letters, vol. 25, no. 2, pp. 133-137, 2012.

[15] X. Li, "Uniform asymptotic stability and global stabiliy of impulsive infinite delay differential equations," Nonlinear Analysis. Theory, Methods \& Applications A, vol. 70, no. 5, pp. 1975-1983, 2009.

[16] X. Li, H. Akca, and X. Fu, "Uniform stability of impulsive infinite delay differential equations with applications to systems with integral impulsive conditions," Applied Mathematics and Computation, vol. 219, no. 14, pp. 7329-7337, 2013. 


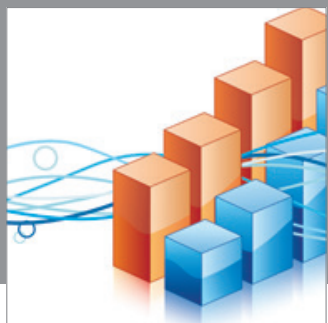

Advances in

Operations Research

mansans

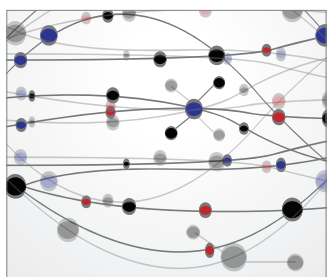

The Scientific World Journal
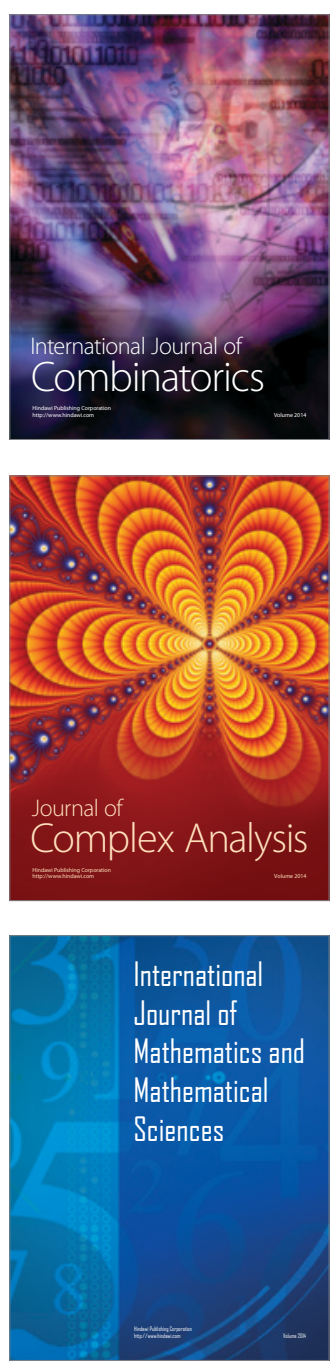
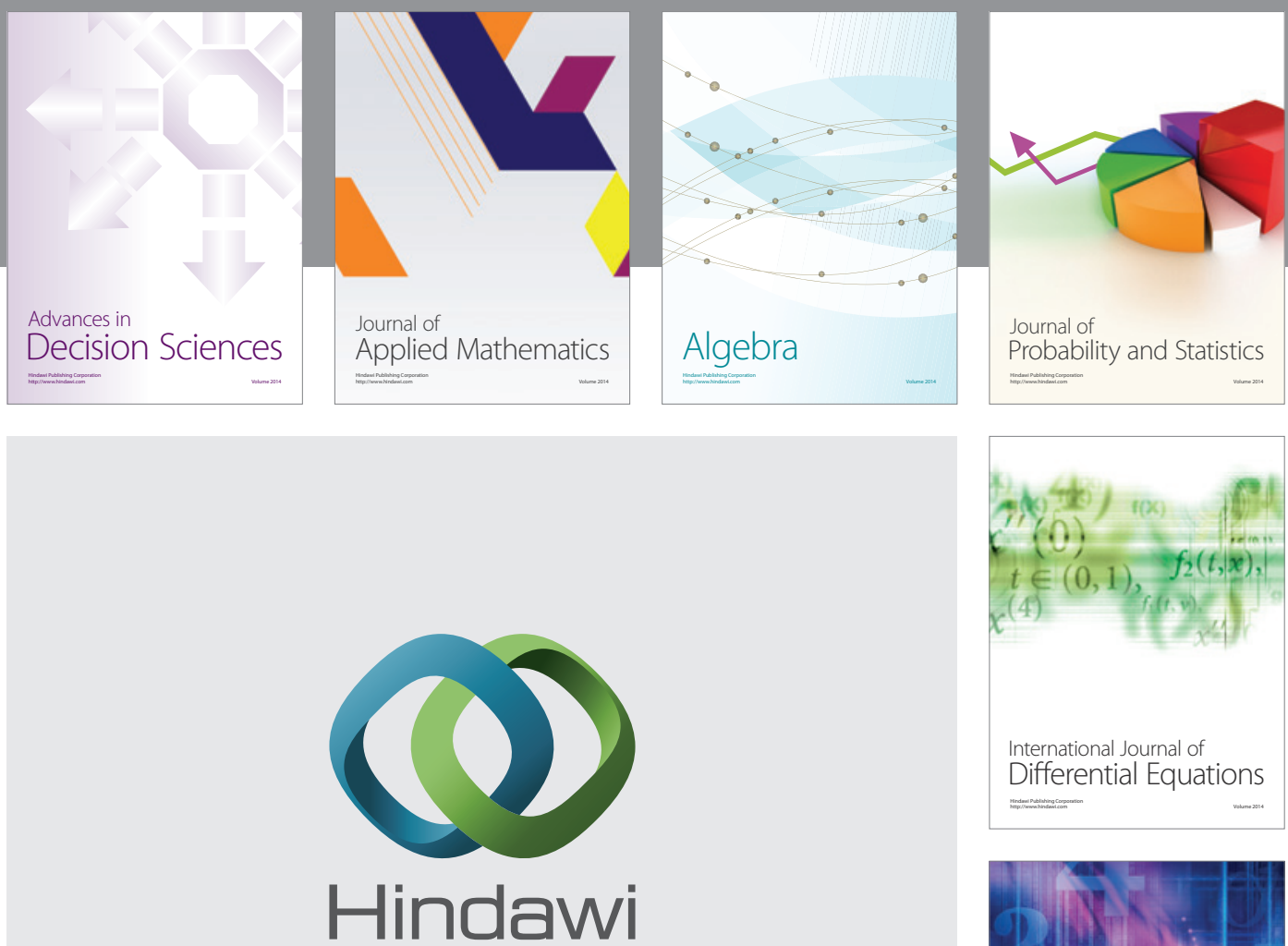

Submit your manuscripts at http://www.hindawi.com
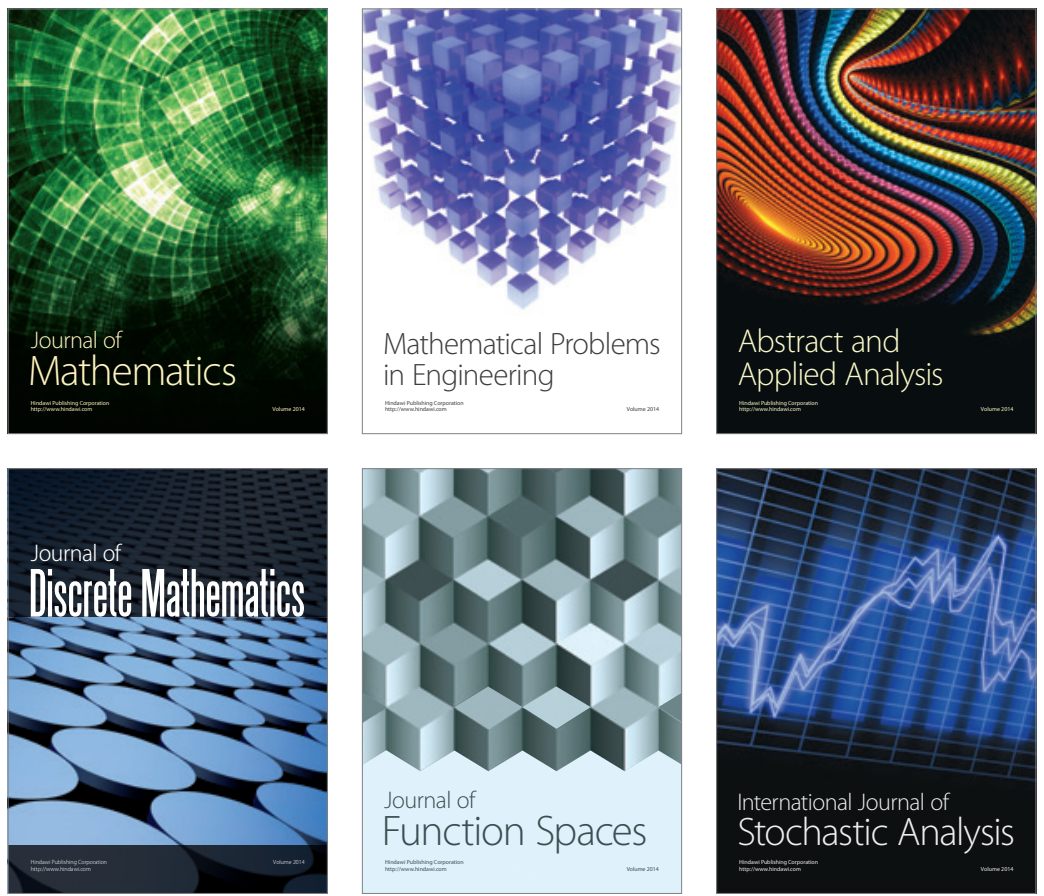

Journal of

Function Spaces

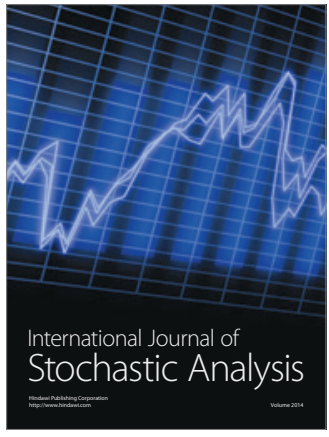

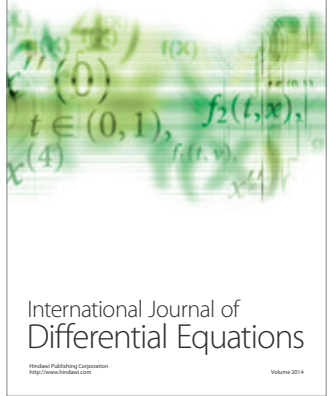
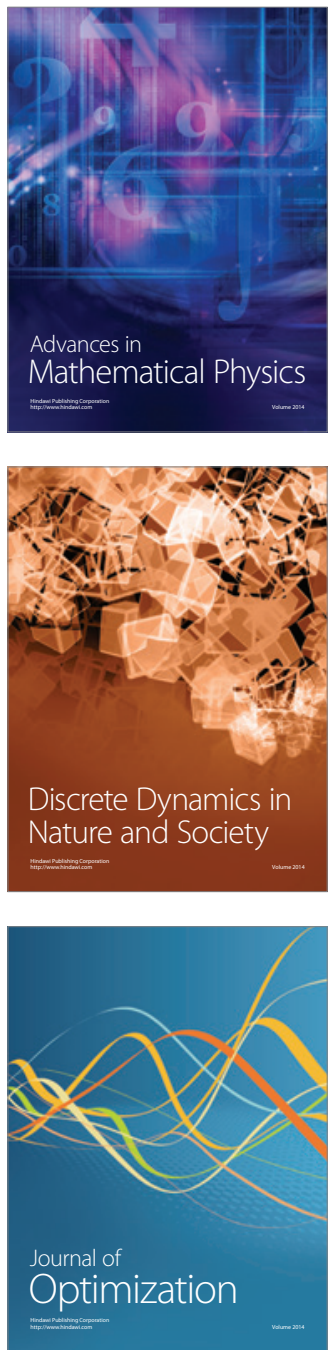